\title{
Balkanologie
}

Balkanologie Revue d'études pluridisciplinaires

Vol. VI, $n^{\circ}$ 1-2 | 2002

Volume VI Numéro 1-2

\section{Force et limites d'une lecture moderne des guerres yougoslaves}

À propos de l'ouvrage Explaining Yugoslavia

Strength and limits of a modern reading of the Yougoslav wars. About John

Allcock's Explaining Yugoslavia

\section{Xavier Bougarel}

\section{OpenEdition}

Journals

Édition électronique

URL : http://journals.openedition.org/balkanologie/459

DOI : $10.4000 /$ balkanologie.459

ISSN : 1965-0582

\section{Éditeur}

Association française d'études sur les Balkans (Afebalk)

\section{Édition imprimée}

Date de publication : 1 décembre 2002

Pagination : 231-244

ISSN : 1279-7952

\section{Référence électronique}

Xavier Bougarel, « Force et limites d'une lecture moderne des querres yougoslaves », Balkanologie [En ligne], Vol. VI, n 1-2 | 2002, mis en ligne le 04 février 2009, consulté le 17 décembre 2020. URL : http:// journals.openedition.org/balkanologie/459; DOI : https://doi.org/10.4000/balkanologie.459 


\section{NOTES ET DOCUMENTS}

NOTES AND DOCUMENTS 


\title{
FORCE ET LIMITES D'UNE LECTURE MODERNE DES GUERRES YOUGOSLAVES À PROPOS DE L'OUVRAGE EXPLAINING YUGOSLAVIA 1
}

\author{
Xavier Bougarel*
}

Dans les premières pages de son ouvrage Explaining Yugoslavia, John Allcock rappelle à juste titre que la plupart des livres consacrés aux récentes guerres yougoslaves " se limitent à fournir un arrière-plan historique, plutôt que de procéder à une analyse historique, et évitent également toute confrontation directe avec des enjeux théoriques " (préface, p. XI). Aspirant au contraire à une sociologie historique de l'espace yougoslave, John Allcock entend " fournir une étude sociologique des transformations historiques de longue durée des sociétés sud-slaves, unies de manière intermittente dans un Etat connu sous le nom de Yougoslavie " (p. 1), et démontrer en particulier que

comprendre la Yougoslavie et ce qu'il est advenu d'elle revient à comprendre des processus dans lesquels nous sommes nous-mêmes impliqués, et à nous confronter comme dans un miroir à nos propres problèmes. (...) La transformation des sociétés balkaniques ne peut trouver de sens que si elle est resituée dans le contexte de ce qu'il advient des sociétés européennes en général (p. 6).

A cette fin, John Allcock présente dans le chapitre deux les concepts-clefs sur lesquels il entend fonder son analyse, à savoir la modernisation et la globalisation, puis explique comment les Balkans participent de longue date à ces deux processus, de manière spécifique mais en relation étroite avec le reste de

\footnotetext{
- Chercheur au CNRS.

${ }^{1}$ Allcock (John), Explaining Yugoslavia, London : Hurst, 2000. Conçu à l'origine comme un simple compterendu d'ouvrage, ce papier a pris peu à peu des dimensions inattendues. En particulier, les développements critiques sur le recours à certains concepts et leur application au cas yougoslave, m'ont demandé une place et un temps considérables. Ce fait ne doit pas être perçu comme une volonté de dévaluer l'ouvrage de John Allcock, mais au contraire comme un hommage implicite à sa richesse, et une mise à nu indirecte de mes propres limites.
} 
l'Europe. Sur cette base, il aborde différents aspects de l'histoire économique, sociale, politique et culturelle de l'espace yougoslave :

a) dans le chapitre trois, il revient d'abord sur l'histoire économique longue des Balkans, et en particulier sur la question des " arriérations " ottomane et austro-hongroise, de leur réalité complexe, de leurs causes internes et externes, de leur perpétuation dans un Royaume de Yougoslavie resté essentiellement rural. Le chapitre quatre traite ensuite de la modernisation communiste, de ses modalités, de ses déséquilibres et de ses contradictions. John Allcock considère qu' " il n'existe pas à l'heure actuelle d'analyse satisfaisante de la dimension économique de l'effondrement de l'Etat yougoslave " (p. 98), et insiste quant à lui sur " une contradiction fondamentale dans l'économie yougoslave entre des impératifs modernisateurs, propres à tout processus d'industrialisation, et des tendances localistes, anti-modernes, inhérentes à son cadre politique " (p. 69). Enfin, le chapitre cinq s'intéresse à la question agraire, à savoir avant tout les structures agraires des Empires ottoman et austro-hongrois, et les réformes agraires des première et deuxième Yougoslavie ;

b) dans le chapitre six, John Allcock déplore que " les questions de démographie aient souffert d'un certain désintérêt au sein de la sociologie " (p. 145), et revient sur les différents mouvements de population qui caractérisent l'histoire de l'espace yougoslave (expansions militaires et migrations économiques, conversions religieuses et assimilations culturelles, exode rural, " nettoyage ethnique ", etc.), ainsi que sur leurs interprétations contemporaines. Le chapitre sept traite de la stratification sociale des sociétés balkaniques : John Allcock s'attache en particulier à montrer comment le caractère segmentaire (localiste et communautariste), semi-périphérique et fortement bureaucratisé de ces sociétés a freiné la constitution des classes bourgeoise et ouvrière associées à la modernité européenne, et conduit plutôt à de simples phénomènes de "massification";

c) le chapitre huit introduit la dimension proprement politique de l'histoire de l'espace yougoslave en retraçant l'émergence et l'évolution des Etats balkaniques, et en insistant sur leur contexte international spécifique (marches des Empires ottoman et austro-hongrois, semi-périphérie capitaliste, division Est-Ouest, etc.). Sur cette base, le chapitre neuf s'intéresse plus spécifiquement à l'échec de la démocratie comme dimension majeure de la modernité politique, et l'explique par la faiblesse historique des institutions représentatives d'une part, la nature populiste et autoritaire de la vie politique dans les première et deuxième Yougoslavie d'autre part. Le chapitre dix prolonge cette analyse en traitant de l'échec de la société civile et du principe de citoyenneté, et renvoie là encore à des tendances historiques longues, à savoir le sous-développement de la société civile, et sa forte communautarisation sur des bases ethniques et religieuses, le communisme autogestionnaire yougoslave n'ayant fait que perpétuer, voire aggraver cet état de fait ; 
d) le chapitre onze amorce le passage des questions politiques aux questions culturelles, en traitant de la constitution des identités nationales, et en insistant sur leur caractère construit et fluctuant, ainsi que sur la diversité des acteurs politiques et culturels impliqués dans l'élaboration de ces " communautés imaginaires " (Benedict Anderson). Le chapitre douze s'interroge, quant à lui, sur le caractère traditionnel et / ou moderne des sociétés balkaniques, et revient en particulier sur la question des structures familiales, des liens d'allégeance clientélistes - l'auteur s'étonnant qu'un phénomène aussi omniprésent et essentiel dans la vie sociale quotidienne ne soit pas systématiquement pris en compte dans la littérature sociologique sur la Yougoslavie (p. 361) -, et des pratiques religieuses. Enfin, le chapitre treize traite de la violence dans les sociétés sud-slaves, en insistant sur la monopolisation tardive et inachevée de la violence légitime par l'Etat, sur l'influence persistante de certaines formes de violence légitime non-étatique (vendetta, banditisme social, etc.), et sur le caractère symbolique et ritualisé de pratiques telles que la mutilation des corps.

John Allcock, spécialiste de longue date de la Yougoslavie, professeur de sociologie et directeur de la Research Unit in South East European Studies à l'université de Bradford (Grande-Bretagne), était sans doute un des mieux à même d'esquisser une sociologie historique de l'espace yougoslave, et d'apporter ainsi un éclairage nouveau sur les guerres yougoslaves. De fait, Explaining Yugoslavia est un livre imposant par son ampleur et son érudition, tout en restant très didactique. Il devrait donc devenir pour longtemps un ouvrage de référence et un outil indispensable pour tous ceux qui étudient l'espace yougoslave. Aux personnes qui travaillent de longue date sur cet espace, il rafraîchira la mémoire et éclaircira l'esprit sur bien des points ; à celles qui le découvre, il en fera découvrir la complexité et la profondeur historique. A ceux qui se réfugient derrière les dates et les noms propres, il montrera que les sciences historiques peuvent avoir d'autres ambitions ; à ceux qui égaient leurs théories sociologiques de références au cas yougoslave, il rappellera que les réalités locales ne se laissent pas facilement domestiquer.

Du point de vue historique, cet ouvrage n'est pas seulement une présentation synthétique et raisonnée de plusieurs siècles d'histoire des pays sud-slaves : il en explore ou en redécouvre certains aspects peu connus ou trop vite oubliés, tels que la nature de la question agraire, de l'" arriération " économique et de la stratification sociale dans l'Empire austro-hongrois, ou encore la place des institutions locales et des établissements bancaires au sein du système autogestionnaire, et l'impact de ce dernier sur la perpétuation des pratiques clientélistes et de l' " esprit de clocher " (" parochialism "), voir sur l'application du principe même de citoyenneté ${ }^{2}$. Les remarques selon lesquelles l'autoges-

\footnotetext{
${ }^{2}$ Voir les développements sur certains aspects méconnus de l'autogestion yougoslave : le « système de délégation $n$ (p. 92), et une de ses conséquences indirectes : le vote multiple de certains citoyens yougoslaves (p. 303).
} 
tion yougoslave aurait été fondamentalement anti-moderne, car favorisant tout à la fois une dédifférenciation institutionnelle et une segmentation verticale de la société (p. 69), et qu'elle aurait non seulement favorisé une républicanisation des économies, mais aussi des formes de "localisme économique [economical parochialism] centré sur la commune et l'entreprise " (p. 77), suscitent bien des réflexions, et méritent d'être prises en compte dans toute réflexion sérieuse sur l'éclatement sanglant de la Yougoslavie.

Ouvrage sociologique, Explaining Yugoslavia permet aussi de replacer l'histoire des pays sud-slaves dans des problématiques plus générales de sciences sociales, et donc d'échapper aux approches évènementielles, moralisantes ou expressément culturalistes des guerres yougoslaves. Chapitre après chapitre, John Allcock revient à ses problematiques centrales que sont la modernisation et la globalisation, en prenant soin de définir les concepts qu'il utilise (" modernisation " et " globalisation ", bien sûr, mais aussi " démocratie ", " société civile ", " citoyenneté ", etc.). Il se réfère non seulement à de grandes figures de l'histoire et de la sociologie (Max Weber, Norbert Elias, Fernand Braudel, Immanuel Wallerstein, Ernest Gellner, Pierre Bourdieu, Antony Giddens, etc.), mais aussi aux meilleurs représentants de la sociologie yougoslave d'après-guerre tels que Josip Županov, Mladen Lazić, Vesna Pešić, Laslo Sekelj ou Vlado Puljiz. Du concept de semi-périphérie emprunté à Wallerstein à celui de civilité inspiré par Elias, John Allcock montre que certains ouvrages classiques de sociologie historique peuvent aider à appréhender des réalités yougoslaves complexes, mais en aucun cas insaisissables. Il nous fait aussi découvrir un certain nombre d'analyses et de débats sociologiques venus de l'espace yougoslave, trop souvent ignorés par les auteurs occidentaux traitant de cette région. Enfin, la bibliographie située en fin de volume donne un bon aperçu de la production scientifique sur l'espace yougoslave parue avant 1990 en langue anglaise et en langue serbo-croate.

Toutefois, cet ouvrage comporte aussi certaines lacunes et, plus largement, a les défauts de ses qualités. Ainsi, en ce qui concerne la bibliographie, on peut s'étonner que certains travaux classiques tels que ceux de Zagorka Golubović sur la crise d'identité de la société yougoslave, ou ceux de Srdjan Vrcan sur la "religion de crise" dans les années 1980 n'y figurent pas 3 . A propos du clientélisme, John Allcock déclare n'avoir " rencontré aucune étude sociale scientifique de ces réseaux [clientélistes] " (p. 363). Ce faisant, il ne tient compte ni des travaux de Josip Županov sur la répartition du pouvoir dans le système autogestionaire4, ou de Žika Minović sur les luttes de pouvoir

\footnotetext{
3 Golubović (Zagorka), Kriza identiteta savremenog jugoslovenskog društva, Belgrade : Filip Višnjić, 1988 ; Vrcan (Srdjan), Od krize religije ka religiji krize, Zagreb : školska knjiga, 1986.
}

4 Županov (Josip), Samoupravljanje i društvena moć, Zagreb : Globus, 1985. 
à l'échelle locale5. De même, sa seule référence relative aux pratiques clientélistes dans les systèmes communistes est le court texte de Ghia Ionescu paru dans l'ouvrage de Gellner et Waterbury ${ }^{6}$, alors que des travaux autrement plus consistants existent sur cette question?.

Mais les vraies limites de l'appareil bibliographique et, au-delà, théorique et conceptuel de l'ouvrage de John Allcock sont ailleurs. D'une part, on peut regretter qu'il ne fasse aucune allusion à l'importante production en langue allemande consacrée à l'espace yougoslave, sans toutefois pouvoir le lui reprocher $^{8}$. D'autre part, il faut noter que son ouvrage est d'une facture très classique et, pour tout dire, relativement datée. Ainsi, s'il donne un aperçu assez complet de la production sociologique yougoslave de la période communiste, il est beaucoup plus lacunaire en ce qui concerne les années de guerre et d'après-guerre9. Il ne tient donc pas compte du fait que l'éclatement de la Yougoslavie et les guerres qui l'ont accompagné ont fortement ébranlé les convictions et les modes de pensée des sociologues (ex-) yougoslaves, jusqu'alors pétris de marxisme et de fonctionnalisme, comme en témoignent certains textes particulièrement émouvants ${ }^{10}$. La production des ethnologues (ex-) yougoslaves, qui ont plus vite su répondre au défi que représentait le surgissement de la violence, est pratiquement passée sous silence, à l'exception du célèbre

${ }^{5}$ Minović (Žika), Politička palanka. Opštinske političke borbe u Srbiji, Beograd : Institut za političke studije, 1972.

${ }^{6}$ Ionescu (Ghia), " Patronage Under Communism n, in Gellner (Ernest), Waterbury (John), eds., Patrons and Clients in Mediterranean Societies, London : Duckworth, 1977.

7 Voir par exemple Rigby (Thomas), Harasymiw (Bohdan), eds., Leadership Selection and Patron-Client Relations in the USSR and Yugoslavia, London : George Allen \& Unwin, 1983 ; Willerton (John), Patronage and Politics in the USSR, Cambridge : Cambridge University Press, 1992; Tarkowski (Jacek), " Patrons and Clients in a Planned Economy n, in Eisenstadt (Shmuel), Lemarchand (René), eds., Political Clientelism, Patronage and Development, London : Sage, 1981; Baker (R.), " Clientelism in the Post-Revolutionary State : the Soviet Union n, in Clapham (Christopher), ed., Private Patronage and Public Power. Political Clientelism in the Modern State, London : Pinter, 1982.

${ }^{8}$ Pour un aperçu synthétique et relativement récent, voir par exemple les bibliographies des ouvrages suivants : Hösch (Edgar), Geschichte der Balkanländer. Von der Frühzeit bis zum Gegenwart, München : C.H. Beck, 1995 ; Hatschikjan (Magarditsch), Troebst (Stefan), Hg., Südosteuropa. Ein Handbuch, München : C. H. Beck, 1999, ainsi que la bibliographie consacrée aux guerres yougoslaves qui se trouve sur le site du Hamburger Institut für Sozialforschung (http://www.his-online.de/projekte/bibliogr.htm).

9 Ainsi, en ce qui concerne les études sociologiques de la société serbe, on peut noter l'absence d'ouvrages tels que Golubović (Zagorka), Kuzmanović (Bora), Vasović (Mirjana), Društveni karakter i društvene promene u svetlu nacionalnih sukoba, Beograd : Institut za filozofiju i društvenu teoriju, 1995 ; Bolčić (Silvano), Društvene promene i svakodnevni život: Srbija početkom devedesetih, Beograd : Institut za sociološka istraživanja, 1995 ; Nemanjić (Miloš), Vuković (Slobodan), Jugoslovensko društvo krajem devedesetih, Beograd : Sociološko društvo Srbije, 1996 ; Samardžić (Slobodan), Nakarada (Radmila), Kovačević (Djuro), Lavirinti krize, Beograd : Institut za evropske studije, 1998 ; Golubović (Zagorka), Stranputice demokratizacije u postsocijalizmu, Beograd : Beogradski krug, 1999.

10 Voir par exemple le dossier "Sociologie et guerre " publiée par la société serbe de sociologie ("Sociologija i rat ", Sociološki pregled, $36(1-4), 1992)$ ou l'introduction écrite par le sociologue croate Josip Županov à son ouvrage Après le déluge (Županov (Josip), Poslije potopa, Zagreb : Globus, 1995). 
ouvrage de Ivan Čolović, Le bordel des guerriers ${ }^{11}$. Des constats similaires pourraient s'appliquer à la littérature scientifique anglo-saxonne à laquelle se réfère John Allcock.

Or, on peut se demander dans quelle mesure il est possible d'analyser les causes et les réalités d'une crise caractéristique des années 1990 à partir d'outils scientifiques datant pour une large part des décennies précédentes. La manière dont John Allcock utilise le concept de globalisation illustre fort bien cette question. Après avoir introduit ce concept en se référant à Giddens, John Allcock privilégie en fait l'approche en termes d'économie-monde et de système mondial élaborée par Wallerstein, et englobe dès lors dans le concept de globalisation des processus datant de plusieurs siècles, tels que l'insertion de l'économie ottomane dans la dite économie-monde, ou la constitution des Etats-nations balkaniques. Par certains aspects, ce choix s'avère utile et salvateur : John Allcock échappe ainsi aux effets de mode liés à la récente " découverte " d'une globalisation qui n'aurait débuté qu'après la chute du mur de Berlin, oppose aux approches culturalistes des " arriérations " ottomane ou balkanique une vision structurelle fondée sur l'opposition centre-périphérie, et rappelle à juste titre que la lecture des réalités balkaniques en termes de sociétés semi-périphériques a donné lieu à des travaux importants et novateurs, quoique eux-mêmes un peu datés ${ }^{12}$.

Il reste que, dans son usage le plus fréquent, le concept de globalisation renvoie à des phénomènes vieux tout au plus de quelques décennies (dérégulation économique et démantèlement de l'Etat-providence, épuisement de l'Etat-nation, émergence chaotique d'une " gouvernance globale ", etc.), et contredisant par certains aspects les processus historiques de plus long terme que John Allcock inclut dans sa propre définition de la globalisation. Dès lors, l'utilisation du concept de globalisation apparait souvent en décalage avec les phénomènes dont traite en réalité John Allcock, et celui-ci s'interdit de comprendre en quoi les guerres yougoslaves sont aussi le produit de la courte durée, ou d'une longue durée qui bascule. Il est significatif, de ce point de vue, que son ouvrage ignore largement les travaux récents liés à l'analyse des sociétés post-communistes ou à la relecture du passé communiste d'une part, aux conflits et aux relations internationales de l'après-guerre froide d'autre part ${ }^{13}$. Or, il eut par exemple été intéressant de comparer le concept de " natio-

\footnotetext{
${ }^{11}$ Colović (Ivan), Bordel ratnika. Folklor, politika i rat, Beograd : XX. Vek, 1994.

12 John Allcock mentionne en particulier les travaux de Ivan Berend, John Lampe et Nicos Mouzelis.

${ }^{13}$ En référence à Michael Ignatieff, John Allcock se déclare " très sceptique sur la validité historique de la thèse d'un type spécifique de "guerre post-moderne" n (p. 409). Pourtant, en s'appuyant sur les travaux de Martin van Creveld (Van Creveld (Martin), The Transformation of War, New York : Free Press, 1991) ou de Mary Kaldor (Kaldor (Mary), New and Old Wars : Organised Violence in the Global Era, Cambridge : Polity Press, 1999), il aurait pu rattacher la problématique des nouveaux conflits à celle du monopole de la violence légitime, qu'il place au coeur de son analyse de la violence dans les sociétés sud-slaves.
} 
nal-féodalisme ", utilisé dès 1988 par Blažo Perović pour caractériser le système yougoslave (p.418) à ceux de " néo-féodalisme " et " néo-médiévalisme ", apparus dans les années 1990, suite aux dérives du post-communisme ${ }^{14}$ et à la multiplication des failed states ${ }^{15}$.

Le fait que l'ouvrage de John Allcock repose sur un appareil scientifique très classique et relativement daté explique, à mon sens, un certain nombre d'approximations et d'erreurs à propos du communisme ou du post-communisme. Ainsi, on peut s'étonner du fait qu'il considère la Constitution de 1974 et la loi de 1976 sur le travail associé comme les vecteurs d'une " repolitisation de la société qu'il n'est pas déraisonnable de décrire comme "stalinienne" " (p. 92), ou d'une " restalinisation du politique " (p.426). En effet, il y a bien eu dans les années 1970 " une confirmation des rigidités structurelles et idéologiques du système, plutôt qu'une avancée réelle vers une démocratie participative " (p. 426), mais cet échec ne s'est en aucun cas accompagné d'un retour à une terreur politique ou à un encadrement idéologique de masse comparables à ceux de la période stalinienne. John Allcock le sait mieux que quiconque, et son recours au terme " stalinisme " trahit donc avant tout une difficulté à conceptualiser les réalités du communisme finissant. Il en va de même quand il estime " difficile d'être certain que le communisme est vraiment mort " (p. 412), vu la force persistante des partis néo-communistes en Pologne, en Russie ou en Serbie, comme si ces partis n'avaient pas eux aussi connu d'importantes mutations, et comme si leurs places dans les jeux politiques polonais, russe et serbe n'étaient pas désormais aussi divergentes qu'inédites. Dans ce contexte, la dénonciation par John Allcock du concept de " transition à la démocratie " (p. 411) apparaît comme une figure de style obligée, et non comme l'amorce d'une réflexion critique sur les analyses dominantes du post-communisme, surtout quand il s'avère que John Allcock attribue la paternité de ce concept à Fukuyama, et non à la " transitologie " apparue dans les années 1970 et 1980 autour des transitions démocratiques - avec ou sans guillemets ? - de l'Europe du sud et de l'Amérique latine.

Le cas de la transition démocratique renvoie à une autre question, à savoir la capacité de John Allcock à dépasser réellement le type d'approches binaires et normatives qu'il dénonce. Ainsi, une des forces de son ouvrage réside dans sa réfutation claire et argumentée des lectures des réalités yougoslaves en termes d'antinomie entre civilisation européenne et barbarie balkanique, moder-

\footnotetext{
14 Voir Verdery (Kathexine), What Was Socialism and What Comes Next ?, Princeton : Princeton University Press, 1996.

15 Voir Cerny (Philip), " Neomedievalism, Civil War and the New Security Dilemma : Globalisation as Durable Disorder n, Civil Wars, 1 (1), Spring 1998. Pour une approche critique de ce type de néologismes, voir Deibert (Ronald), " Exorcismus Theoriae : Pragmatism, Metaphors and the return of the Medieval in International Relations Theory ", European Journal of International relations, 3 (2), 1997.
} 
nité austro-hongroise et arriération ottomane, balkanisation de l'Europe et européanisation des Balkans. Mais la manière dont, par exemple, John Allcock appréhende la question du nationalisme dans l'espace yougoslave n'est ellemême pas exempte d'oppositions binaires et de présupposés normatifs.

Dans le clivage fondateur qui oppose les analyses primordialistes et constructivistes du phénomène national, John Allcock se situe clairement du côté des secondes, comme l'atteste sa référence appuyée à Benedict Anderson. Il peut dès lors, à juste titre, réfuter les billevesées sur le caractère artificiel des nations macédonienne ou musulmane, en soulignant que, si " le processus de construction [nationale] est dans ces deux cas plus récent, et donc plus aisément perceptible, il n'a pas un caractère fondamentalement différent de celui des autres nations " (p. 315). Mais on se demande alors ce qu'il veut dire quand il affirme que " les diverses nations de l'espace yougoslave sont devenues et sont des nations de manières différentes, et les conséquences de ces différents types de communautés nationales et de formes de la conscience nationale varient donc considérablement " (p. 313), ou que les guerres yougoslaves " mettent en jeu différents types de communauté, qui ont développé différents types de discours politique ", et dont les " types de revendication et les modes discursifs qui les modèlent sont incommensurables " (p. 314).

S'il s'agissait simplement d'insister sur l'historicité propre à chaque nation sud-slave, et de préciser dès lors leurs trajectoires historiques respectives, ces remarques seraient fort bienvenues ${ }^{16}$. Mais, comme beaucoup d'autres auteurs, John Allcock distingue en fait sans nuances deux types de nationalisme - le nationalisme civique et le nationalisme ethnique - , et fonde sur cette opposition son analyse de la question nationale dans l'espace yougoslave. Or, si les concepts de nationalisme civique et nationalisme ethnique peuvent, en tant qu'idéal-types, avoir certaines vertus heuristiques, leur utilisation pour classer les nationalismes réellement existants de l'espace yougoslave conduit John Allcock à oublier leurs ambivalences, leurs oscillations et leurs clivages internes, à commettre certaines erreurs d'interprétation et, finalement, à passer à côté des vrais questions théoriques que soulève à ce sujet l'histoire mouvementée de l'espace yougoslave.

Sur le plan factuel, John Allcock tend ainsi à considérer la réalité des divers nationalismes de l'espace yougoslave au cours des années 1990 à travers le seul prisme des partis nationalistes dominants. Or, au sein de chaque communauté nationale, différents acteurs ont en fait cherché à se poser en représentant légitime de l'idéologie nationale, et en ont offert des définitions divergentes. En outre, il faudrait se demander si les partis dominants qu'ont été le SPS en Serbie, le HDZ en Croatie ou le SDA dans la communauté musulmane bos-

${ }^{16}$ Notons toutefois qu'elles forceraient peut-être John Allcock à nuancer, ou à préciser les formes de son adhésion aux thèses constructivistes. 
niaque étaient soudés par une définition précise et stable de la nation ou, au contraire, par un discours polysémique et fluctuant qui les dispensait de choisir entre plusieurs définitions concurrentes. Quand, par exemple, John Allcock considère que l'erreur des dirigeants serbes est d'avoir " opté pour le principe ethnique " (p. 428), il oublie que ces derniers ont aussi joué la carte de l'idée yougoslave, en 1988 contre les dirigeants slovènes, en 1990 contre Vuk Drašković, en 1994 contre Radovan Karadžić ou en 1999 contre les nationalistes albanais et l'OTAN ${ }^{17}$.

Sur le plan conceptuel, John Allcock décrit l'opposition nationalisme civique / nationalisme ethnique en termes d'" opposition entre l'ethnos et le demos - ou entre une "communauté imaginaire" (...) définie soit en termes d'identité ethnique partagée, soit de citoyenneté commune " (p. 428). Or, comme l'a bien montré Ernest Gellner ${ }^{18}$, l'essence même de tout projet nationaliste est de vouloir constituer tout à la fois une identité culturelle et une communauté politique communes - la nation -, et de les faire coïncider au sein d'un Etat moderne et territorial - l'Etat-nation. Dès lors, les différences et les clivages qui apparaissent entre nationalismes portent plutôt sur les moyens qu'ils se donnent pour atteindre ces objectifs (assimilation, expulsion ou extermination), et sur les rythmes et les modes selon lesquels s'articulent les différentes dimensions d'un seul et même processus.

Alors que les guerres yougoslaves peuvent être l'occasion d'un retour critique sur les concepts de nation et de citoyenneté ${ }^{19}$, ou sur les violences et les apories constitutives de leur mise en œuvre ${ }^{20}$, il est dommage que John Allcock ramène finalement la question nationale dans l'espace yougoslave à une opposition statique et réifiée entre nationalisme civique et nationalisme ethnique. Cela est d'autant plus vrai que, par ce biais, il cède lui-même à la tentation des hiérarchies normatives. C'est le cas quand, pour des raisons peu convaincantes, il estime que le projet nationaliste des Serbes et des Croates " continuera de constituer un obstacle à leur accession à la modernité ", quand celui des Bochniaques, des Monténégrins, des Macédoniens ou des Albanais du Kosovo " ne laisse apparaître aucune contradiction directe avec la modernité " (p. 349). C'est également le cas quand, après avoir confondu projet identitaire bochniaque (" bošnjak ) et projet multiethnique bosniaque - soit, en ses pro-

${ }^{17}$ On peut se demander, il est vrai, si l'idée yougoslave elle-même constitue un nationalisme civique ou un nationalisme ethnique (Jugo-sloven : Slave du sud).

${ }^{18}$ Gellner (Ernest), Nations and Nationalism, Oxford : Blackwell, 1983.

19 Voir par exemple Gourgouris (Stathis), " Enlightenment and Paranomia ", in De Vries (Hent), Weber (Samuel), eds., Violence, Identity and Self-Determination, Stanford : Stanford University Press, 1997.

20 Voir par exemple Hayden (Robert), " Imagined Communities and Real Victims : Self-Determination and Ethnic Cleansing in Yugoslavia n, American Ethnologist, 23 (4), novembre 1996. 
pres termes, projet ethnique et projet civique -, il déclare que ce dernier est " pratiquement le seul représentant de l'idée européenne d'un Etat basé sur une identité civique et non ethnique " (p. 336). Cela revient en effet à identifier l'Europe au (bon) nationalisme civique, et les Balkans au (mauvais) nationalisme ethnique, et donc à réintroduire dans l'analyse cette antinomie Europe / Balkans que John Allcock s'efforce par ailleurs de réfuter.

C'est là, sans doute, que se rejoignent les questions du (non-dépassement de certains présupposés normatifs d'une part, du recours à un appareil scientifique de facture classique d'autre part. Plus que celui de globalisation, en effet, c'est le concept de modernisation qui supporte l'ensemble de l'ouvrage Explaining Yugoslavia. A cette fin, John Allcock en reprend la définition donnée par Jürgen Habermas ${ }^{21}$, avant de préciser que la modernisation est " un processus complexe qui se déroule sur plusieurs dimensions - économique, politique et symbolique ", qu'il existe une " multiplicité de modes possibles d'insertion du pré-moderne dans le moderne ", et que "la modernisation ne doit plus être conceptualisée en termes de remplacement d'une culture pré-moderne par une culture moderne, mais plutôt d'une rencontre avec la modernité, dans laquelle les ressources des sociétés pré-modernes sont déployées afin d'adapter, et même de s'approprier certains éléments de la modernité " (p. 17 et 18). Il met donc en garde contre certains usages du couple modernité / tradition, et note ainsi avec pertinence que « la frontière entre la ville et la campagne n'est pas le point limite d'expansion de la modernité, mais une zone de transition entre deux aspects de la modernisation " (p. 8), que les Empires ottoman et austro-hongrois " ne doivent pas être considérés simplement comme des obstacles au processus de modernisation, mais comme les biais par lesquels la modernité acquiert une forme, un rythme et une orientation spécifiques " (p. 11), et que " la région des Balkans dans son ensemble a bien été marquée par une certaine arriération, en termes de rythme et de niveau d'industrialisation ", mais que " sous d'autres aspects, toutefois, il faut reconnaître l'ampleur relative de son implication dans la modernité, et de sa pénétration par cette dernière " (p. 17).

Par contre, si " la tradition est une ressource qui permet aux gens de s'adapter aux conditions changeantes de la modernité " (p. 11), on voit mal le fondement théorique de sa distinction entre partis nationalistes modernisateurs et partis nationalistes traditionnalistes qui, par son caractère statique et nor-

\footnotetext{
${ }^{21}$ " Le concept de modernisation désigne un ensemble de processus cumulatifs qui se renforcent les uns les autres ; il désigne la capitalisation et la mobilisation des ressources, le développement des forces productives et l'augmentation de la productivité du travail ; il désigne également la mise en place de pouvoirs politiques centralisés et la formation d'identités nationales ; il désigne encore la propagation des droits à la participation politique, des formes de vie urbaine et de l'instruction publique ; il désigne enfin la laïcisation des valeurs et des normes "(Habermas, (Jürgen), Le discours philosophique de la modernité, Paris : Gallimard, 1988, pp. 2, 3).
} 
matif, évoque celle établie précédemment entre nationalisme civique et nationalisme ethnique. Du reste, appliquée aux cas serbe et macédonien, cette grille de lecture donne des résultats fort décevants. John Allcock écrit que le Parti socialiste serbe " est traditionnaliste sous de nombreux aspects, quoi que nationaliste ", et s'oppose aux partis albanais qui, "bien qu'étant eux aussi nationalistes, sont dans l'ensemble une force modernisatrice " (p. 438). Or, s'il existe un clivage entre traditionnalistes et modernisateurs, il traverse les nationalismes serbe et albanais plutôt qu'il n'oppose l'un à l'autre. De plus, si les partis albanais du Kosovo doivent être considérés comme modernisateurs, c'est dans la mesure même où ils sont nationalistes, et cherchent à ériger en communauté nationale une population albanaise dont les allégeances restent dans une large mesure familiales, régionales et religieuses ${ }^{22}$. De même, quand John Allcock voit dans la coalition entre les nationalistes du VMRO macédonien et ceux du PDPSH albanais une alliance de deux partis porteurs d'un projet de modernisation économique, il confond discours électoraux et pratiques de pouvoir, et oublie le rôle des prébendes, des réseaux clientélistes et des clivages générationnels dans la vie politique macédonienne ${ }^{23}$.

On peut donc, là encore, se demander si John Allcock ne retombe pas parfois dans les travers développementalistes ou culturalistes qu'il dénonce. En reliant son analyse de la société civile à l'opposition nationalisme ethnique / nationalisme civique, il confond par exemple deux problèmes distincts, à savoir la faiblesse réelle ou supposée de la société civile dans l'espace yougoslave, et sa fragmentation selon des lignes de rupture ethniques et religieuses d'autre part. Pourtant, certaines sociétés civiles florissantes ont été ou restent pétries de communautarisme, comme l'atteste l'exemple des Pays-Bas et des Etats-Unis. Par la suite, John Allcock a beau préciser que « la civilité n'est pas nécessairement un mode de comportement moralement supérieur ", et que la problématique qui lui est liée " ne peut pas être réduite au contraste entre anciennes régions habsbourgeoises et ottomanes " (p. 305), il n'en finit pas moins par considérer que, dans l'espace yougoslave, le recours au " piston " ("veze ") est l'apanage des régions post-ottomanes, ce qui reste à démontrer.

L'usage que John Allcock fait du concept de civilité pose donc lui aussi un certain nombre de problèmes pratiques. Quand il voit dans l'" incivilité " de la vie politique serbe " un trait autochtone, et non un héritage "turc" " (p. 306), il tend à projeter la réalité des années 1990 sur l'ensemble de l'histoire politique

\footnotetext{
${ }^{22}$ Pour savoir si les partis nationalistes serbes sont ou non modernisateurs, il faudrait alors se demander si les Serbes sont quant à eux pleinement constitués comme communauté nationale, ou encore dominés par un certain nombre d'allégeances primaires, puis, dans un second temps, sur lesquels de ces éléments les partis réputés nationalistes assoient en fait leur influence.

${ }^{23}$ Sur le plan idéologique, le clivage entre SDSM et PDP d'une part, VMRO et PDPSH d'autre part est moins un clivage entre traditionnalistes et modernisateurs qu'entre néo-communistes et anti-communistes, ce qui n'est pas forcément la même chose.
} 
moderne de la Serbie ${ }^{24}$. Il oublie également que la disparition des élites politiques médiévales serbes peut être considérée comme une conséquence de l'expansion ottomane, et que d'autres élites serbes ont pu perdurer ou apparaître dans les siècles suivants, tant en Serbie étroite que dans l'Empire austrohongrois (Voïvodine, Dalmatie, etc.) ou dans des villes bosniaques telles que Sarajevo et Mostar ${ }^{25}$. Enfin, voir dans la Bosnie-Herzégovine, et dans Sarajevo en particulier, l'unique dépositaire de la civilité ottomane ${ }^{26}$, c'est oublier d'une part que l'espace yougoslave englobe d'autres centres urbains importants de l'époque ottomane, tels que Skoplje ou Prizren, d'autre part que les populations rurales bosniaques sont associées à la civilité urbaine par le biais du čaršija, comme l'a bien montré William Lockwood dans les années $1970^{27}$, et possèdent leurs propres formes de civilité, décrites plus récemment par Tone Bringa ${ }^{28}$.

John Allcock n'est donc pas insensible aux catégories et hiérarchies normatives produites par divers protagonistes des guerres yougoslaves ${ }^{29}$, et n'échappe pas toujours à certains travers développementalistes ou culturalistes. Sur ce second point, les ambiguités de John Allcock sont de toute évidence liées à son appareil scientifique, les grandes figures auxquelles il se réfère comptant parmi les principaux artisans de la mise en forme théorique de la modernité occidentale. Or, à la définition classique de la société civile qu'utilise John Allcock, il serait par exemple possible d'opposer Chris Hann et Elisabeth Dunn,

\footnotetext{
24 Au contraire, les historiens serbes ont eu tendance dans les années 1990 à redécouvrir la période parlementaire de la Serbie indépendante, et à s'interroger à travers elles sur les contradictions et les aléas du processus de modernisation politique en Serbie. Voir par exemple Perović (Latinka), Obradović (Marija), Stojanović (Dubravaka), ur., Srbija u modernizacijskim procesima XX. veka, Beograd : INIS, 1994 ; Popović (Olga), Parlamentarizam u Srbiji od 1903. do 1914. godine, Beograd : Službení list SRJ, 1998.

25 Sur Sarajevo, voir Skarić (Vladislav), "Srpski pravoslavni narod i crkva u Sarajevu u 17. i 18. vijeku ", in Izabrana djela - knjiga II : prilozi o istoriji Sarajeva, Sarajevo : Veselin Masleša, 1985.

${ }^{26}$ A propos du cas bosniaque, John Allcock parle pas de civilité (post-)ottomane, mais de " traditions autochtones islamiques de civilité " (p. 305). Or, l'idée selon laquelle la civilité bosniaque serait l'apanage de l'islam, et donc de la seule communauté musulmane, alors même qu'elle est censée permettre " l'interaction (...) entre des individus issus de milieux divers " (p. 305), est un dangereux contresens - la prétention au monopole de la tolérance tue la tolérance - , et une simple projection sur l'histoire de la Bosnie-Herzégovine de certains clichés nationalistes réactivés par les guerres yougoslaves.
}

27 Lockwood (William), European Moslems. Economy and Ethnicity in Western Bosnia, NewYork / London : Academic Press, 1974.

${ }^{28}$ Bringa (Tone), Being Muslim the Bosnian Way. Identity and Community in a Central Bosnian Village, Princeton : Princeton University Press, 1995.

29 L'anthropologue Marko Žikvović a bien montré comment la dénonciation du " manque de culture " était dans les années 1990 un des thèmes récurrents des élites politiques et intellectuelles serbes, tant nationalistes qu'anti-nationalistes (Živković (Marko), "Too Much Character, Too Little Kultur : Serbian Jeremiads 1994-1995 ", Balkanologie, 2 (2), décembre 1998). Par ailleurs, les nationalistes serbes euxmêmes n'hésitent pas à se revendiquer à leur manière du " processus de civilisation ", en s'enorgueillissant du fait que les empereurs serbes utilisaient des fourchettes, à une époque où les souverains occidentaux mangeaient encore avec leurs doigts ! 
qui cherchent à en dépasser l'ethnocentrisme ${ }^{30}$, au " processus de civilisation " repris de Elias, d'opposer Hans Peter Duerr, pour qui celui-ci n'est qu'un mythe occidental ${ }^{11}$, et ainsi de suite.

Faute d'avoir la capacité de se livrer à cette tâche immense, on s'étonnera simplement du fait que, au terme d'un ouvrage construit de bout en bout autour de l'idée de modernité, et dont une des dimensions implicites reste la valorisation de ses principaux attributs politiques, surgissent deux grandes figures de sa déconstruction post-moderne, à savoir Michel Foucault et Zygmunt Bauman. C'est bien sûr la violence des guerres yougoslaves, et les interrogations qu'elle traîne dans son sillage, qui amène John Allcock à mentionner ces deux auteurs. Mais leur apparition tardive, soit tombe à plat, soit fait vaciller l'ensemble de l'ouvrage : faut-il en effet rappeler que Foucault conteste la pertinence même du concept de société civile, et parle de dressage des corps plutôt que de polissage des mœurs ${ }^{32}$, ou que Bauman voit lui aussi dans le processus de civilisation un "mythe étiologique profondément ancré dans la conscience de soi de notre société occidentale "33 ?

Dans ce contexte, il n'est pas surprenant que le chapitre final consacré à la violence dans les sociétés sud-slaves soit à la fois le plus prometteur et le plus décevant de tous. Dans ses considérations sur les formes légitimes de violence dans les sociétés sud-slaves, ou sur les dimensions symboliques et rituelles de certaines atrocités, John Allcock montre une fois de plus l'étendue de son érudition. Dans ses mises en garde sur le traitement des crimes qui ont accompagné les guerres yougoslaves, il fait aussi preuve d'un grand courage intellectuel. Mais force est de constater qu'il ne parvient pas à relier la question de la violence dans l'espace yougoslave à sa problématique centrale de la modernisation.

Certes, en insistant sur le caractère tardif et inachevé de la monopolisation de la violence légitime par l'Etat, et donc de la différenciation entre une société pacifiée et un appareil militaire spécialisé, John Allcock met l'accent sur un aspect essentiel de l'histoire des sociétés sud-slaves. Mais son usage flou et quelque peu étrange du concept de militarisation ne permet pas de savoir, par exemple, dans quelle mesure le système yougoslave de défense populaire généralisé relevait d'une persistance diffuse de formes de violence légitime non-

${ }^{30}$ Hann (Chris), Dunn (Elisabeth), eds., Civil Society : Challenging Western Models, London : Routledge, 1996.

${ }^{31}$ Duerr (Hans Peter), Nudité et pudeur. Le mythe du processus de civilisation, Paris : éditions de la Maison des sciences de l'homme, 1998.

32 Foucault (Michel), "Il faut défendre la société ". Cours au Collège de France 1976, Paris : Gallimard / Seuil, 1997 ; Foucault (Michel), Surveiller et punir, Paris : Gallimard, 1975.

33 Bauman (Zygmunt), Legislators and Interpreters, Cambridge : Polity Press (1987), p. 12, cité dans Smith (Dennis), Zygmunt Bauman, Prophet of Postmodernity, Cambridge : Polity Press, 1999, p. 226. 
étatique, ou de l'emprise insidieuse sur la société d'un appareil militaire posttotalitaire. De même, en qualifiant les pratiques de vendetta, le culte des bandits sociaux et les violences ritualisées de "dimensions de cette militarisation [non-pacification] résiduelle des cultures sud-slaves " (p. 388), il ne tient pas compte de leur réinvention par l'Etat modernisateur, et retombe ainsi dans un usage antinomique du couple tradition / modernité.

Dès lors, il lui est difficile d'appréhender les liens existants entre modernisation et violence des guerres yougoslaves : le meurtre du voisin est ainsi expliqué par la persistance des codes de la vendetta, et non par la diffusion du principe de citoyenneté qui, loin de prolonger la civilité post-ottomane, en annexe les mécanismes et les brise ${ }^{34}$. Au delà, la modernité des guerres yougoslaves se trouve finalement ramenée à quelques indices mineurs (reprise de symboles guerriers issus de l'imaginaire occidental, utilisation de l'artillerie dans le siège des villes, etc.), eux-mêmes rejetés parmi les formes " aberrantes " de la violence, comme si John Allcock percevait confusément que la proximité entre modernité et violence - révélée, entre autres, par Michel Foucault et Zygmunt Bauman - était l'élément pouvant remettre en cause l'ensemble de sa propre construction intellectuelle.

Dans ce contexte, ses remarques éparses sur les violences et les crimes propres au monde occidental ne lui permettent en aucun cas d'atteindre son objectif de départ, à savoir " percevoir la nature fondamentalement problématique du capitalisme et de l'identité européenne, et reconnaitre comme problématique le projet politique démocratique dans son ensemble " et, finalement, utiliser l'espace yougoslave pour " nous confronter comme dans un miroir à nos propres problèmes " (p. 6). En effet, ce que John Allcock problématise avec force, c'est moins l'essence de la modernité que sa possibilité d'existence au sein de l'espace yougoslave. Son ouvrage mesure les réalités sud-slaves à l'aune du projet occidental, et non l'inverse : en d'autres termes, il montre ce que la sociologie nous apprend de la Yougoslavie, mais pas ce que la Yougoslavie pourrait nous apprendre sur la sociologie.

34 Je me permets sur ce point de renvoyer au chapitre trois ( $\mathrm{Du}$ bon voisinage au crime intime ") de mon livre Bosnie, anatomie d'un conflit, Paris : La Découverte, 1996. 